\title{
Front Matter: Volume 11283
}

, "Front Matter: Volume 11283," Proc. SPIE 11283, Integrated Optics: Devices, Materials, and Technologies XXIV, 1128301 (27 March 2020); doi:

$10.1117 / 12.2567572$

SPIE. Event: SPIE OPTO, 2020, San Francisco, California, United States 


\section{PROCEEDINGS OF SPIE}

\section{Integrated Optics: Devices, Materials, and Technologies XXIV}

Sonia M. García-Blanco

Pavel Cheben

Editors

3-6 February 2020

San Francisco, California, United States

Sponsored and Published by

SPIE

Volume 11283 
The papers in this volume were part of the technical conference cited on the cover and title page. Papers were selected and subject to review by the editors and conference program committee. Some conference presentations may not be available for publication. Additional papers and presentation recordings may be available online in the SPIE Digital Library at SPIEDigitalLibrary.org.

The papers reflect the work and thoughts of the authors and are published herein as submitted. The publisher is not responsible for the validity of the information or for any outcomes resulting from reliance thereon.

Please use the following format to cite material from these proceedings:

Author(s), "Title of Paper," in Integrated Optics: Devices, Materials, and Technologies XXIV, edited by Sonia M. García-Blanco, Pavel Cheben, Proceedings of SPIE Vol. 11283 (SPIE, Bellingham, WA, 2020) Seven-digit Article CID Number.

ISSN: 0277-786X

ISSN: 1996-756X (electronic)

ISBN: 9781510633292

ISBN: 9781510633308 (electronic)

Published by

SPIE

P.O. Box 10, Bellingham, Washington 98227-0010 USA

Telephone +1 3606763290 (Pacific Time) · Fax +1 3606471445

SPIE.org

Copyright (c) 2020, Society of Photo-Optical Instrumentation Engineers.

Copying of material in this book for internal or personal use, or for the internal or personal use of specific clients, beyond the fair use provisions granted by the U.S. Copyright Law is authorized by SPIE subject to payment of copying fees. The Transactional Reporting Service base fee for this volume is $\$ 21.00$ per article (or portion thereof), which should be paid directly to the Copyright Clearance Center (CCC), 222 Rosewood Drive, Danvers, MA 01923. Payment may also be made electronically through CCC Online at copyright.com. Other copying for republication, resale, advertising or promotion, or any form of systematic or multiple reproduction of any material in this book is prohibited except with permission in writing from the publisher. The CCC fee code is $0277-$ $786 \times / 20 / \$ 21.00$.

Printed in the United States of America by Curran Associates, Inc., under license from SPIE.

Publication of record for individual papers is online in the SPIE Digital Library.

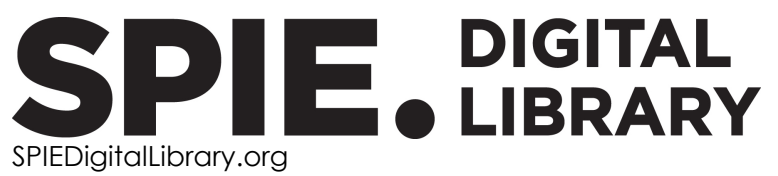

Paper Numbering: Proceedings of SPIE follow an e-First publication model. A unique citation identifier (CID) number is assigned to each article at the time of publication. Utilization of CIDs allows articles to be fully citable as soon as they are published online, and connects the same identifier to all online and print versions of the publication. SPIE uses a seven-digit CID article numbering system structured as follows:

- The first five digits correspond to the SPIE volume number.

- The last two digits indicate publication order within the volume using a Base 36 numbering system employing both numerals and letters. These two-number sets start with $00,01,02,03,04$, 05, 06, 07, 08, 09, 0A, OB ... 0Z, followed by 10-1Z, 20-2Z, etc. The CID Number appears on each page of the manuscript. 


\section{Contents}

$\begin{array}{ll}\text { vii } & \text { Authors } \\ \text { ix } & \text { Conference Committee }\end{array}$

INVERSE DESIGN OF INTEGRATED PHOTONIC CIRCUITS I

1128305 Deep-learning-assisted on-chip Fourier transform spectrometer [11283-3]

NOVEL MATERIALS AND PLATFORMS

$11283 \mathrm{OB} \quad$ Seeing blue: pushing integrated photonics into the ultraviolet with ALD aluminum oxide (Invited Paper) [1 1283-9]

11283 OC Low loss $\mathrm{TiO}_{2}$ channel waveguides [11283-10]

PHOTONIC INTEGRATION TECHNOLOGIES

$11283 \mathrm{OH} \quad$ InP grating coupler design for vertical coupling of InP and silicon chips [1 1283-15]

11283 0J Hybrid integration of a polarization independent optical circulator [1 1283-17]

OPTICAL SENSORS I

11283 OL High-order PT symmetry-based optical sensor [1 1283-19]

$11283 \mathrm{OM}$ Suspended silicon waveguide for mid-infrared gas sensing [11283-20]

OPTICAL SENSORS II

11283 OP CMOS-compatible silicon nitride waveguide photonic building blocks and their application for optical coherence tomography and other sensing applications (Invited Paper) [1 1283-23]

$112830 Q \quad$ Ultra-sensitive photonic integrated circuit-based biosensors for healthcare applications (Invited Paper) [1 1283-24]

11283 OR Highly sensitive silicon Mach-Zehnder interferometer-based ultrasound sensor [11283-25] 
11283 OS Coupling condition engineered subwavelength grating waveguide ring resonator for sensitivity enhancement [11283-26]

PHOTONIC INTEGRATED CIRCUITS

$112830 \mathrm{X}$ Silicon photonic phase interrogators for on-chip calibration of optical phased arrays [11283-31]

11283 OY 28 Gbps silicon-germanium hetero-structure avalanche photodetectors [11283-32]

PROGRAMMABLE INTEGRATED PHOTONICS

1128311 Low-power reconfigurable photonic integrated circuits fabricated by femtosecond laser micromachining [11283-35]

ARTIFICIAL INTELLIGENCE WITH INTEGRATED OPTICS

1128313 A chaotic microresonator structure for an optical implementation of an artificial neural network (Invited Paper) [1 1283-37]

NONLINEAR PHOTONICS

1128319 Enhancement of third-order nonlinearity of thermally evaporated GeSbSe waveguides through annealing [11283-43]

OPTICAL SENSORS III

11283 IE Fabrication of multichannel Bloch long-range surface plasmon biosensors [1 1283-48]

OPTICAL SENSORS IV

$112831 \mathrm{G}$ Optimizing resolution in an integrated blazed chirped Bragg grating spectrometer [1 1283-50]

$11283 \mathrm{lH}$ Thermo-optically tuned spatial heterodyne Fourier-transform spectrometer [11283-51]

$1128311 \quad$ Silicon nitride waveguide platform for on-chip spectroscopy at visible and NIR wavelengths [11283-52]

$112831 \mathrm{~J} \quad$ Alloy-based wire array metamaterial fibres and hyperlenses for imaging applications at MIR frequencies [11283-53] 
$112831 \mathrm{~K} \quad$ Design and fabrication of multilayer GRIN lenses by multi-material additive manufacturing for light coupling applications in planar optoelectronic systems [1 1283-54]

\section{POSTER SESSION}

1128310 A fluorescence spectroscopy biosensor for lab-on-a-chip detection of antibiotics in milk [11283-58]

$112831 \mathrm{P}$ Techniques to achieve low series resistance, high photon absorption rate, and high quantum efficiency for photonic CMOS field effect transistors [1 1283-59]

$112831 \mathrm{IT} \quad$ Analysis of additive manufactured polymer optical waveguides: measurement and simulation of their waviness [1 1283-63]

$112831 \mathrm{U}$ Optimization of solder reflow processing and part design in thermoplastic optical interconnect components [11283-64]

$112831 \mathrm{~V}$ Integrated high-resolution and broad-bandwidth optical spectrum analyzer [11283-65]

11283 IW Optofluidic device for sorting microparticles using optical whispering gallery mode resonances [11283-66]

$112831 \mathrm{~A} \quad$ Athermal chirp-compensated directly modulated PIC for uncooled DWDM [1 1283-67]

1128312 A scalable fibre optic sensing architecture for lab-on-a-chip devices [11283-69]

1128320 Design and analysis of polarization beam splitter based on cascaded MMI on SOI [1 1283-70]

1128322 Waveguides sensitivity analysis for mid-infrared gas sensing [11283-72]

$1128323 \quad$ Fabrication of optical waveguide using a non-contact printing technique [11283-73]

1128327 The integrated vertically coupled resistive random-access memory (ReRAM)-based microdisk resonator and the relevant performance evaluation [11283-77]

1128328 The impact of ultraviolet light on the switching characteristics of NiO resistive random-access memory (ReRAM) devices [1 1283-78]

$1128329 \quad$ Aerosol jet printed PZT actuated MEMS resonating cantilever scanner [1 1283-79]

$112832 F \quad$ Foundry-compatible thin-film lithium niobate electro-optic modulators [1 1283-85]

$112832 \mathrm{G}$ Beam finite spot size effect on angle-tolerant optical filters [11283-86] 
$112832 \mathrm{H} \quad$ Geometry optimization of unidirectional integrated ring laser [11283-87]

Proc. of SPIE Vol. $112831128301-6$

Downloaded From: https://www.spiedigitallibrary.org/conference-proceedings-of-spie on 25 Apr 2023 Terms of Use: https://www.spiedigitallibrary.org/terms-of-use 


\title{
Authors
}

Numbers in the index correspond to the last two digits of the seven-digit citation identifier (CID) article numbering system used in Proceedings of SPIE. The first five digits reflect the volume number. Base 36 numbering is employed for the last two digits and indicates the order of articles within the volume. Numbers start with 00, 01, 02, 03, 04, 05, 06, 07, 08, 09, OA, OB...0Z, followed by 10-1Z, 20-2Z, etc.

\author{
Alonso-Ramos, Carlos, $\mathrm{OY}, 1 \mathrm{H}$ \\ Amar, Farah, OY \\ Anandarajah, Prince M., IX \\ Andersson, Sean B., IW \\ Anous, Noha, 2G \\ Atzeni, S., 11 \\ Aubin, Guy, OY \\ Backhaus, C., IT \\ Bae, Kyuyoung, 19 \\ Baets, Roel, OR \\ Ballabio, A., $1 \mathrm{H}$ \\ Bannerman, Rex H. S., $1 G$ \\ Bardella, Paolo, $2 \mathrm{H}$ \\ Barzaghi, A., 1H \\ Baudot, Charles, OY \\ Benedikovic, Daniel, OY \\ Benson, Trevor M., 13 \\ Berini, Pierre, 1E \\ Berry, Sam A., $1 G$ \\ Bienstman, Peter, 13 \\ Boeuf, Frédéric, OY \\ Bosma, Rick, 10 \\ Bouville, D., $1 \mathrm{H}$ \\ Braddell, Jules, IX \\ Caro, Jacob, OR \\ Cassan, Eric, OY \\ Ceccarelli, F., 11 \\ Chack, Devendra, 20 \\ Chakravarty, Swapnajit, 2F \\ Chang, Yuhe, IW \\ Chen, Peng-Jyun, 29 \\ Choi, Young-Wan, 23 \\ Chuang, Ricky W., 27, 28 \\ Collier, Christopher M., 10, 12 \\ Conradi, Hauke, OJ \\ Crespi, A., 11 \\ Crozat, Paul, OY \\ Dahlem, Marcus S., OX \\ de Felipe, David, OJ \\ Deniel, L., $1 \mathrm{H}$ \\ Devasagayam, Jasen, 10 \\ Dijkstra, M., OC \\ Donegan, John F., IX \\ Drexler, Wolfgang, OP \\ Dwivedi, Sarvagya, OX \\ Eggeling, Moritz, OP \\ Eiche, Y., $1 \mathrm{~T}$ \\ Ekinci, Kamil, $1 \mathrm{~W}$ \\ El Shamy, Raghi S., OM, 22
}

Falke, Floris, $O Q$

Fédéli, Jean-Marc, OY

Field, James W., IG

Figeys, Bruno, OX

Franke, J., $1 \mathrm{~T}$

Frigerio, J., $1 \mathrm{H}$

Fu, Kuan-Lun, 27

Gali, Sushma, 11

García-Blanco, S. M., OC

Gates, James C., $1 \mathrm{G}$

Gawith, Corin B. E., 1G

Geuzebroek, Douwe H., OQ

Ghillino, Enrico, $2 \mathrm{H}$

Giannuzzi, Giuseppe, 2H

Gopinath, Juliet T., 19

Gotoda, Mitsunobu, $\mathrm{OH}$

Grayson, Michael, 19

Grigoleto Hayashi, Juliano, $1 \mathrm{~J}$

Gutierrez-Pascual, Deseada, IX

Hainberger, Rainer, OP

Hartmann, Jean-Michel, OY

Hassan, Shamsul, 20

Hayashi, Shusaku, $\mathrm{OH}$

Hegeman, I., OC

Heideman, René G., $O Q$

Hirbodvash, Zohreh, IE

Hoffmann, G. A., IT

Hohenhoff, Gerrit, 1K

Hoogland, Gabrie, $1 \mathrm{U}$

Horsten, Roland, OR

Hsu, Yi-Feng, 29

Hu, You-Kui, 28

Huang, Jinhua, OP

Huang, Ming-Cheng, 28

Isella, G., 1H

Ismail, Dima, $1 \mathrm{Z}$

Jaeschke, Peter, $1 \mathrm{~K}$

Jaffar, Noor, $1 \mathrm{Z}$

Jain, Gaurav, $1 \mathrm{X}$

Janicek, Petr, $1 \mathrm{~J}$

Jansen, Roelof, OX

Jiang, Ke-Jian, $O P$

Johnson, Peter M., $1 \mathrm{U}$

Jordan, Nathan J., IW

Kaierle, Stefan, $1 \mathrm{~K}$

Keil, Norbert, 0J

Kerman, Sarp, OX

Khalil, Diaa A., OM, 22, 2 G

Khodami, Maryam, $1 \mathrm{E}$ 
Kim, Hyeon Beom, 23

Kim, Hyungchan, 23

King, Alexander S., IW

Kjellman, Jon Øyvind, OX

Klamkin, Jonathan, $\mathrm{OH}$

Kleinert, Moritz, OJ

Ko, Hyun, 23

Ko, Jeong Beom, 23

Koike-Akino, Toshiaki, $\mathrm{OH}$

Kojima, Keisuke, $\mathrm{OH}$

Kongnyuy, Tangla David, OX

Kopp, Christophe, OY

Kraft, Jochen, OP

Kresse, Martin, OJ

Kruidhof, Marten, OR

Krupin, Oleksiy, $1 \mathrm{E}$

Kumar Tyagi, Hemant, OX

Kumar, Abhishek, 20

Leclerc, Camille A., $1 \mathrm{Z}$

Leinse, Arne, $O Q$

Le Roux, Xavier, OY, $1 \mathrm{H}$

Li, Ming-Yao, 29

Li, Mingzhu, OP

Li, Ting, 05, OL, OS

$L i$, Yanlu, OR

Lindlein, N., IT

Lisicka-Skrzek, Ewa, 1E

Liu, Q., $1 \mathrm{H}$

Maese-Novo, Alejandro, OP

Marinins, Aleksandrs, OX

Marris-Morini, Delphine, $\mathrm{OY}, \mathrm{IH}$

McKenna, Robert, 1X

Meissner, Thomas, $\mathrm{OH}$

Mellor, Chris, 13

Misra, Arijit, 1V

Montesinos-Ballester, M., $1 \mathrm{H}$

Muellner, Paul, OP

Nevlacsil, Stefan, OP

Nishikawa, Satoshi, $\mathrm{OH}$

Northfield, Howard, IE

Nuck, Madeleine, oJ

Oh, Geum-Yoon, 23

Ohno, Norihiko, $1 \mathrm{U}$

Olivieri, Anthony, 1E

Osellame, R., 11

Ouyang, Boling, OR

Overmeyer, Ludger, 1K, IT

Pan, James N., IP

Park, Wounjhang, 19

Parsons, Kieran, $\mathrm{OH}$

Pellegatta, F., 11

Peng, Kuan-Cheng, 29

Pentangelo, C., 11

Phang, Sendy, 13

Poletti, Francesco, 1J

Preußler, Stefan, IV

Prost, Mathias, OX

Raghunathan, Varun, 11

Ramirez, J.-M., $1 \mathrm{H}$

Rank, Elisabet, OP
Reitberger, T., $1 \mathrm{~T}$

Rezaei, Hossein S., $1 \mathrm{~K}$

Rottenberg, Xavier, OX

Safian, Reza, 2F

Sagmeister, Martin, OP

Sang, Fengqiao, $\mathrm{OH}$

Schell, Martin, OJ

Scheres, LUC, OQ

Scheu, Anja, OJ

Schneider, Thomas, $1 \mathrm{~V}$

Schotter, Joerg, OP

Schreuder, Frederik, $0 Q$

Schuetz-Trilling, Anke, $O Q$

Seddon, Angela, 13

Selvaraja, Shankar Kumar, 11

Seyringer, Dana, OP

Singh, Ashutosh, 10

Smith, Peter G. R., $1 G$

Smyth, Frank, $1 \mathrm{X}$

Song, Bowen, $\mathrm{OH}$

Song, Yanlin, OP

Soussan, Philippe, OX

Spotts, Isaac, 12

Sugawara, Takamune, $1 \mathrm{U}$

Svitelskiy, Oleksiy, IW

Swillam, Mohamed A., OM, 22

Szelag, Bertrand, OY

Tang, Yingheng, $\mathrm{OH}$

Teng, Min, 2F

Troia, Benedetto, OX

Tsui, Chi-Leung, 29

Vakarin, V., 1H

van der Meer, Adriaan, $\mathrm{OQ}$

van Dongen, Koen W. A., OR

Vestering-Stenger, Rachel, $O Q$

Virot, Léopold, OY

Vivien, Laurent, OY, $1 \mathrm{H}$

Vogelbacher, Florian, OP

Wan, Weishi, OL

Wang, Wei-Chih, 29

West, Gavin N., OB

White, Nicholas, $1 \mathrm{~J}$

Wu, Wen-Jong, 29

Wu, Yulin, OL

Xia, Lipeng, 05

$\mathrm{Yi}$, Xiongsheng, $\mathrm{OH}$

Zawadzki, Crispin, 0J

Zergioti, loanna, $0 Q$

Zhang, Aoxue, 05

Zheng, Zhe-Ya, 27, 28

Zhou, Linjie, $1 \mathrm{~V}$

Zhou, Peiji, OL

Zhou, Xue, OP

Zhu, Jiangang, 19

Zhuang, Leimeng, $2 \mathrm{~F}$

Zohrabi, Mo, 19

ZOU, Yi, 05, OL, OS 


\section{Conference Committee}

Symposium Chairs

Sailing He, KTH Royal Institute of Technology (Sweden) and Zhejiang University (China)

Yasuhiro Koike, Keio University (Japan)

Symposium Co-chairs

Connie J. Chang-Hasnain, University of California, Berkeley (United States)

Graham T. Reed, Optoelectronics Research Center, University of Southampton (United Kingdom)

Program Track Chairs

Yakov Sidorin, Quarles \& Brady LLP (United States)

Jean-Emmanuel Broquin, IMEP-LAHC (France)

Conference Chairs

Sonia M. García-Blanco, Universiteit Twente (Netherlands)

Pavel Cheben, National Research Council Canada (Canada)

Conference Program Committee

Pierre Berini, University of Ottawa (Canada)

Romeo Bernini, Istituto per il Rilevamento Elettromagnetico dell'Ambiente (Italy)

Andrea Blanco Redondo, The University of Sydney (Australia)

Alexandra Boltasseva, Purdue University (United States)

Jean-Emmanuel Broquin, IMEP-LAHC (France)

Florenta A. Costache, Fraunhofer-Institut für Photonische Mikrosysteme

IPMS (Germany)

Xudong Fan, University of Michigan (United States)

Robert Halir, Universidad de Málaga (Spain)

Gualtiero Nunzi Conti, Istituto di Fisica Applicata Nello Carrara (Italy)

Alessia Pasquazi, University of Sussex (United Kingdom)

François Royer, Université Jean Monnet Saint-Etienne (France)

Jens H. Schmid, National Research Council Canada (Canada)

Yakov Sidorin, Quarles \& Brady LLP (United States)

Winnie N. Ye, Carleton University (Canada)

Avinoam Zadok, Bar-llan University (Israel) 
Wei Zhou, Virginia Polytechnic Institute and State University (United States)

\section{Session Chairs}

1 Inverse Design of Integrated Photonic Circuits I

Sonia M. García-Blanco, Universiteit Twente (Netherlands)

2 Inverse Design of Integrated Photonic Circuits II

Iñigo Molina-Fernández, Universidad de Málaga (Spain)

3 Novel Materials and Platforms

Jean-Emmanuel Broquin, IMEP-LAHC (France)

4 Photonic Integration Technologies

Rainer Hainberger, AIT Austrian Institute of Technology GmbH (Austria)

5 Optical Sensors I

Carlos A. Alonso-Ramos, Centre de Nanosciences et de Nanotechnologies (France)

6 Optomechanics

Florenta A. Costache, Fraunhofer-Institut für Photonische Mikrosysteme IPMS (Germany)

7 Optical Sensors II

Sonia M. García-Blanco, Universiteit Twente (Netherlands)

8 Photonic Integrated Circuits

Pavel Cheben, National Research Council Canada (Canada)

9 Programmable Integrated Photonics

Yakov Sidorin, Quarles \& Brady LLP (United States)

10 Artificial Intelligence with Integrated Optics

Jean-Emmanuel Broquin, Institut de Microélectronique Électromagnétisme et Photonique et le Lab d'hyperfrequences et Caracte (France)

11 Nonlinear Photonics

Joan Manel Ramírez, III-V Laboratory (France)

12 Optical Sensors III

Jean-Emmanuel Broquin, IMEP-LAHC (France) 
13 Optical Sensors IV

Florenta A. Costache, Fraunhofer-Institut für Photonische Mikrosysteme IPMS (Germany)

14 Photonic Devices

Jens H. Schmid, National Research Council Canada (Canada)

Proc. of SPIE Vol. $112831128301-11$

Downloaded From: https://www.spiedigitallibrary.org/conference-proceedings-of-spie on 25 Apr 2023 Terms of Use: https://www.spiedigitallibrary.org/terms-of-use 
Proc. of SPIE Vol. 11283 1128301-12

Downloaded From: https://www.spiedigitallibrary.org/conference-proceedings-of-spie on 25 Apr 2023 Terms of Use: https://www.spiedigitallibrary.org/terms-of-use 